\title{
Prevalence of intestinal parasitic infection among primary school children in southern Iran
}

\author{
Habibollah Turki ${ }^{1}$ Yaghoob Hamedi ${ }^{1}$ Mehregan Heidari-Hengami ${ }^{1}$ • \\ Majid Najafi-Asl $^{1} \cdot$ Soroush Rafati $^{2} \cdot$ Khojasteh Sharifi-Sarasiabi $^{1}$
}

Received: 28 August 2016/Accepted: 3 November 2016/Published online: 23 November 2016

(C) Indian Society for Parasitology 2016

\begin{abstract}
A cross-sectional study was carried out to determine the prevalence of intestinal parasites in primary school children in Bandar Abbas, southern Iran during January to March 2016. Single stool samples were collected from 1465 participants by clean stool cup. The questionnaire was prepared on the basis of demographic characteristics. The diagnosis was made on the basis of the direct wet mount, formalin-ether concentration, and ZiehlNeelsen and trichrome stained slides. Out of the 1465 school children examined, six species of intestinal parasites were identified with an overall prevalence of 95 positive students $(6.5 \%)$. Prevalence of protozoan infections (6.2\%) was significantly higher than helminth infections $(0.3 \%)$. The most common protozoan species were Giardia lamblia $(n=42,2.9 \%)$ and Blastocystis hominis $(n=31,2.1 \%)$. Only two cases of Hymenolepis nana and two cases of Enterobius vermicularis were detected. The prevalence of parasitic infections was higher in boys $(n=52,7 \%)$ than girls $(n=41,5.7 \%$ ), but the difference was not significant. The parasitic infections were higher among the children whose parent's education was less than high school diploma $(p=0.000)$, and there was also a significant association between the parents' jobs and the intensity of parasitic infection. The prevalence of intestinal parasites, compared to the previous studies in this area, was significantly lower; that is mainly due to an increase in the quality
\end{abstract}

Khojasteh Sharifi-Sarasiabi

sharifisarasiabi@gmail.com

1 Infectious and Tropical Diseases Research Center, Hormozgan University of Medical Sciences, Bandar Abbas, Iran

2 Student Research Committee, Hormozgan University of Medical Sciences, Bandar Abbas, Iran of life and parents' education as well as the accessibility to health services. The most prevalent intestinal parasite was G. lamblia; therefore, it is recommended that more emphasis is applied to controlling and preventing this parasitic infection by washing hands, particularly before each meal course.

Keywords Prevalence · Intestinal parasites ·

Primary school children

\section{Introduction}

Intestinal parasites are transmitted directly by water, soil, and/or food contaminated with fecal materials or indirectly by inappropriate personal and social hygiene conditions (Daryani et al. 2012). The high prevalence of intestinal parasites in children is associated with many factors, especially personal and environmental hygiene (Taheri et al. 2011). Intestinal parasites reduce absorption of dietary supplies and other nutritious materials, induce intestinal blood loss, and destroy red blood cells by splenic filtration that results in anemia in children (Mahmud 2015; Moore et al. 2015). Infections with helminths (e.g., Ascaris lumbricoides, hookworms, Hymenolepis nana, and Trichuris trichiura) and intestinal protozoa (e.g., Entamoeba histolytica and Giardia lamblia) are directly associated with poverty, contaminated water, inadequate medical care, and inappropriate health and environmental status (Matthys et al. 2011; Sah et al. 2013). Therefore, the distribution and frequency of intestinal parasites are different from one area to another (Ayalew et al. 2011). The rate of intestinal parasite infection is a useful index, especially in undeveloped and developing countries. Such infections may lead to malnutrition and inadequate physical growth which cause 
undesirable consequences in learning and cognitive ability, especially in school children (Warunee et al. 2007; Fallahi et al. 2016).

In a study in Ethiopia, the prevalence of intestinal parasites among school children was $34.2 \%$ (Gelaw et al. 2013). In another study in Nigeria, $28 \%$ of studied children were infected with one or more intestinal parasites (Gimba and Dawam 2015). In another survey that was conducted on students in eastern Afghanistan (Korzeniewski et al. 2015), $39.1 \%$ of the participants were infected with nematodes, cestodes, trematodes, and intestinal protozoa. The prevalence of intestinal parasites is different among students from different regions of Iran; e.g., in Lorestan it was reported to be 11.93\% (Fallahi et al. 2016); in Sari, northern Iran, it was 33.3\% (Daryani et al. 2012); and in Golestan, northern Iran, it was $28.8 \%$ (Masoumeh et al. 2012). In order to control and prevent this infectious disease, it is necessary to perform periodic surveys to estimate the prevalence of intestinal parasites in different populations (Taheri et al. 2011).

The present study aimed to determine the prevalence of intestinal parasites in primary school children of Bandar Abbas, southern Iran, and its association with demographic characteristics. A similar study was carried out 14 years ago (Sharifi Sarasiabi et al. 2002) in this area. Thus, the present study was designed to examine the trend of intestinal parasitic infection among the school children during this period.

\section{Materials and methods}

\section{Study population and sample collection}

In this cross-sectional study, 1465 stool samples were collected from students attending 30 primary schools of Bandar Abbas, Hormozgan province in a 3-month period from January to March 2016. The province is located in the south of Iran, in a tropical region attached to the Persian Gulf with high humidity (20-100\%) and a warm climate (Shokri et al. 2012). Bandar Abbas is situated on a flat area with an average altitude of $9 \mathrm{~m}$ (30 ft.) above sea level and a population of approximately 450,000 people. (https://en.Wikipedia.org/wiki/BandarAbbas). A simple random sampling method was employed to select both schools and children from each area (west and east of Bandar Abbas) using a table of random numbers. Then, the students were given questionnaires for completion by their parents (mother or father) or legal guardians. The questionnaire was prepared on the basis of age, gender, educational grades, district of education and training (west and east), as well as the parents' occupation and level of education. Furthermore, all the participants were asked to answer the question about the antiparasitic drugs they already took; children who were taking antiparasitic drugs during the data collection or those who refused to participate were excluded from the study (Fig. 1). All the children used a city water supply and a toilet for defecation. A single stool sample was collected from each participant with a clean stool cup. Each container was labeled and immediately transferred to the laboratory of the paramedical faculty of Hormozgan University of Medical Sciences (HUMS). The diagnosis was made on the basis of the direct wet mount (normal saline and lugol), formalin-ether concentration method, and Ziehl-Neelsen and trichrome stained slides.

\section{Statistical analysis}

The collected data were analyzed using SPSS software version 20. Descriptive statistics were used to assess the prevalence of intestinal parasites while the relationship between demographic factors and presence of intestinal parasites was determined by the Chi-squared test. The level of significance was 0.05 .

The study protocol was approved by the Infectious and Tropical Diseases Research Center, HUMS, Bandar Abbas, Iran. Ethics approval for the project (HUMS. REC 1394.30) was obtained from the Ethics Committee of the HUMS.

The objectives of the study were explained to the officials of the schools, the health practitioners, and the students. In addition, written informed consent was obtained from all the participants' parents or the legal guardians before sample collection.

\section{Results}

The study included 741 (50.6\%) boys and 724 (49.4\%) girls out of 1465 children who were examined for intestinal parasitic infections. The mean age of the study subject was 9.2 years with a minimum and maximum age of 6 and 14 years, respectively. Since there were few students of a given age, we decided to categorize them into two groups to obtain optimum analysis. According to age level, the distribution rate was 791 aged less than 9 years $(54 \%)$ and 674 aged at least 9 years $(46 \%)$. Diarrhea was present in $11(0.8 \%)$ of the subjects, none of whom reported to have abdominal pain or any intestinal parasites.

The prevalence of intestinal parasites in the west area of Bandar Abbas (6.8\%) was more than in the east (6.04\%), but the difference was not significant. 


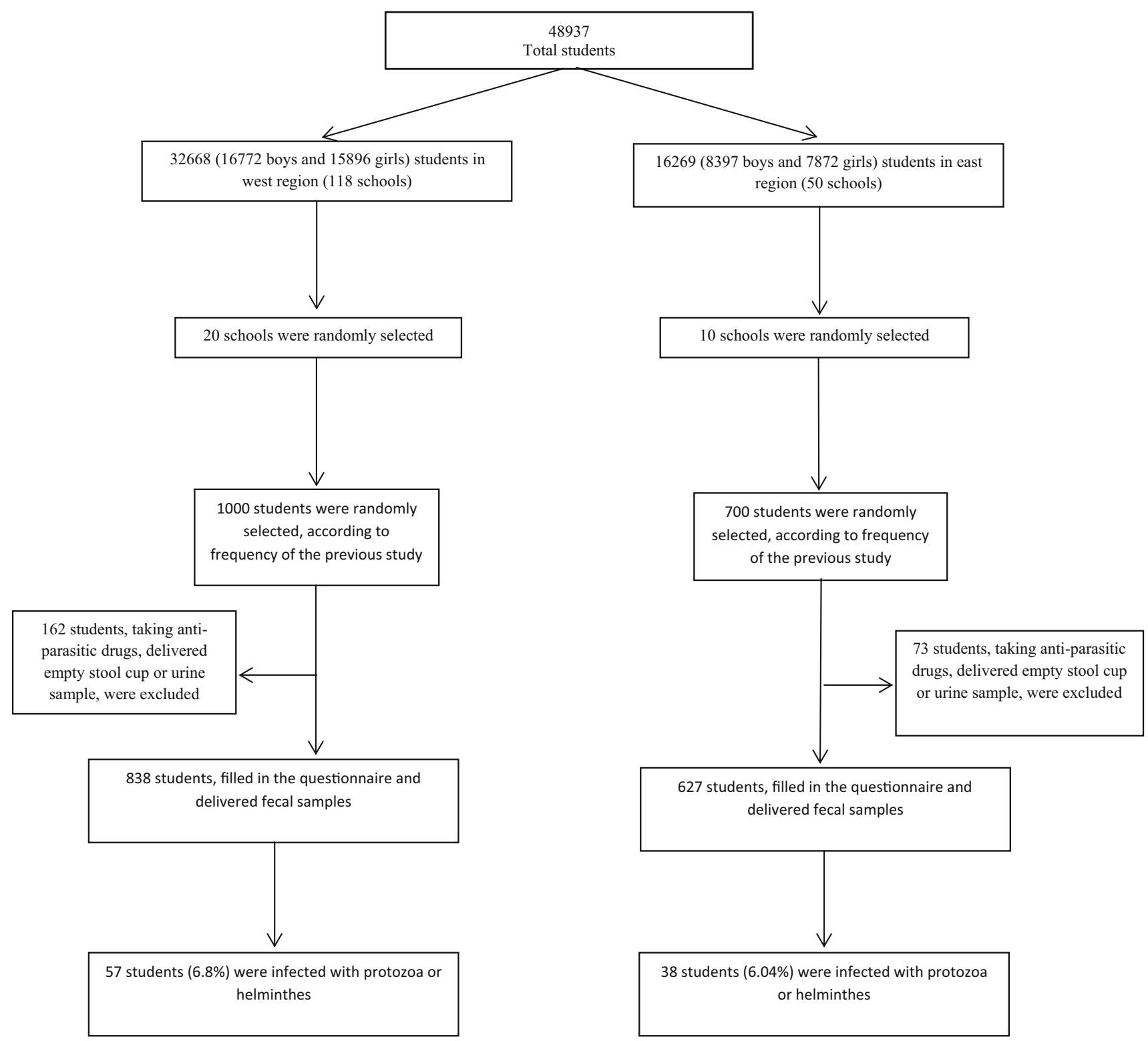

Fig. 1 Flow chart of 1465 students of primary schools of Bandar Abbas, southern Iran to determine the prevalence of intestinal parasites, in a 3-month period from January to March 2016

Most of the students' mothers, 728 (49.7\%), had a high school diploma, and 277 (18.9\%) had an academic degree (Table 1).

Out of the 1465 school children examined, six species of intestinal parasites were identified with an overall prevalence of 95 positive students $(6.5 \%)$. Prevalence of protozoan infections $(6.2 \%)$ was significantly higher than helminth infections $(0.3 \%)$. As shown in Table 2 , the most common protozoan species were G. lamblia $(n=42$, $2.9 \%)$ and Blastocystis hominis $(n=31,2.1 \%)$.

Of the total $95(6.5 \%)$ positive individuals only $2(2.1 \%)$ showed double infection (one with G. lamblia and B. ho- minis and the other with E. coli and B. hominis) and the rest had a single infection. The prevalence of parasitic infections was higher in boys $(n=54,7 \%)$ than in girls ( $n=41,5.7 \%$ ); however, the difference was not significant. The parasitic infections were higher among children whose parents' education level was below a high school diploma than those whose parents had at least a high school diploma (Table 1). Furthermore, direct microscopy was compared with formalin-ether concentration method in 1465 samples. Direct microscopy was positive in 57 (3.9\%) of the samples. The use of formalin-ether concentration increased the number of positive samples to 95 (6.5\%). 
Table 1 Association between sociodemographic characteristics and parasitic infections among primary school children in southern Iran

\begin{tabular}{|c|c|c|c|c|}
\hline Sociodemographic characteristics & Number of students (\%) & Positive (\%) & OR & $P$ value \\
\hline \multicolumn{5}{|l|}{ Gender } \\
\hline Boys & $741(50.6)$ & $54(7)$ & $1.257[0.824-1.919]$ & 0.335 \\
\hline Girls & $724(49.4)$ & $41(5.7)$ & & \\
\hline \multicolumn{5}{|l|}{ Age group } \\
\hline$<9$ & $791(54)$ & $53(6.7)$ & $1.138[0.745-1.739]$ & 0.312 \\
\hline$\geq 9$ & $674(46)$ & $42(5.9)$ & & \\
\hline \multicolumn{5}{|l|}{ Area } \\
\hline East area & $627(42.8)$ & $38(6.04)$ & $0.850[0.552-1.307]$ & 0.264 \\
\hline West area & $838(57.2)$ & $57(6.8)$ & & \\
\hline \multicolumn{5}{|l|}{ Level of education (grade) } \\
\hline $1-3$ & $791(54)$ & $57(7.2)$ & $1.312[0.854-2.014]$ & 0.128 \\
\hline $4-6$ & $674(46)$ & $38(5.6)$ & & \\
\hline \multicolumn{5}{|l|}{ Mother's occupation } \\
\hline Housewife & $1267(86.5)$ & $89(7)$ & $2.881[1.155-7.185]$ & \\
\hline Practitioner & $198(13.5)$ & $6(3)$ & & 0.008 \\
\hline \multicolumn{5}{|l|}{ Mother's education } \\
\hline Before diploma & $728(49.7)$ & $63(8.7)$ & & 0.000 \\
\hline Diploma & $460(31.4)$ & $27(5.9)$ & & \\
\hline Academic degree & $277(18.9)$ & $5(1.8)$ & & \\
\hline \multicolumn{5}{|l|}{ Father's occupation } \\
\hline Employed & $537(36)$ & $23(4.4)$ & & 0.007 \\
\hline Worker & $437(29.8)$ & $41(9.4)$ & & \\
\hline Self-employed & $501(34.2)$ & $31(6.2)$ & & \\
\hline \multicolumn{5}{|l|}{ Father's education } \\
\hline Before diploma & $632(43.1)$ & $63(10)$ & & 0.000 \\
\hline Diploma & $513(35)$ & $25(4.9)$ & & \\
\hline Academic degree & $320(21.8)$ & $7(2.2)$ & & \\
\hline
\end{tabular}

Table 2 Distribution of parasitic infection among primary school children in southern Iran

\begin{tabular}{lcl}
\hline Intestinal parasites & Frequency & Percentage \\
\hline Positive & 95 & 6.5 \\
Helminths & 4 & 0.3 \\
Protozoa & 91 & 6.2 \\
No infection & 1370 & 93.5 \\
Total & 1465 & 100 \\
Name of parasites & & \\
G. lamblia & 42 & 2.9 \\
B. hominis & 31 & 2.1 \\
E. coli & 17 & 1.2 \\
C. mesnili & 1 & 0.1 \\
H. nana & 2 & 0.1 \\
E. vermicularis & 2 & 0.1 \\
\hline
\end{tabular}

\section{Discussion}

The current study showed a prevalence of $6.5 \%$ for intestinal parasites by various methods, including direct microscopic observation through normal saline and Lugol, the formalin-ether concentration method, and stained by the Ziehl-Neelsen and trichrome methods in 1465 students of Bandar Abbas schools. This prevalence was much less than in the previous study (48.4\%) (Sharifi Sarasiabi et al. 2002).

Only six species were found in this study, which showed less diversity of parasitic species in this area. Low soil moisture and limited herbal coverage are considered the most important reasons for the lower prevalence of intestinal parasites and their diversity as a result of the remarkable reduction of rainfall in recent years; other factors are drinking healthy water, severe sunlight, the 
avoidance of using human stool as fertilizer, and a higher environmental hygiene level, especially the use of asphalt for streets and alleys. Furthermore, families' tendencies to consume homemade foods, as opposed to fast foods, especially for the children, might be another reason for the decrease of intestinal parasites. However, the direct transmitted parasites such as G. lamblia, E. coli, or B. hominis are still high in the region. Studies conducted in school students in Turkey (Okyay et al. 2004) and Afghanistan (Korzeniewski et al. 2015), Iran's neighbors, showed that the prevalence of intestinal parasites was $31.8 \%$ and $39.1 \%$, respectively, which were higher than in our study.

Generally, it seems that the prevalence of intestinal parasites, especially intestinal helminths and in particular some intestinal nematodes, has decreased in the last decade in Iran (Rokni 2008). Low prevalence of intestinal helminthiasis $(0.3 \%)$ in the present study, compared with the previous study (Sharifi Sarasiabi et al. 2002) (5.91\%), supports this claim. The high prevalence of soil-transmitted helminths, such as A. lumbricoides, in other studies, like those in Ethiopia and Angola (Ayalew et al. 2011; Oliveira et al. 2015), are probably due to the low living standards and environmental health, as well as people's lack of awareness of the sanitary basics (Ayalew et al. 2011). Washing and cooking of vegetables, wearing shoes, supervision of children's play areas, and proper facilities for the disposal of sewage as well as using the toilet for fecal defecation, drinking healthy water, and living in urban areas are protecting factors against many parasites, particularly parasites that are transmitted through soil, such as A. lumbricoides, hookworms, Strongyloides stercoralis, and G. lamblia (Jejaw et al. 2015).

The conditions of samples examined in this study were the same as in the previous study, since the subjects all used urban water and toilets for fecal defecation, and lived in cities. Therefore, it is concluded that improvement of personal and environmental hygiene, using chemical soil fertilizer, and increased levels of awareness and literacy of the society have been very effective.

The prevalence of $H$. nana, in comparison with other parasitic helminths was somewhat higher in Iran. However, there seems to be a downward trend since the 1970s (Rokni 2008). According to our findings, the prevalence of H. nana was $0.1 \%$. Nevertheless, in the previous study (Sharifi Sarasiabi et al. 2002), the prevalence of this parasite was $4.3 \%$. Recently, the distribution of fast foods or snacks is forbidden in primary schools, and soaps have become available for handwashing; hence, these factors could have contributed to the observed prevalence reduction. The prevalence of intestinal parasites has been reported to be $6.7 \%$ in South Khorasan province in east of Iran (Taheri et al. 2011), $1.5 \%$ in Golestan province in northern Iran, and $25.8 \%$ in western Tajikistan, our northern neighbor (Matthys et al. 2011).

The prevalence of E. vermicularis was $0.1 \%$ in the present study, which was consistent with the results of the study by Al-Mohammed et al. in Saudi Arabia (Al-Mohammed et al. 2010) and Daryani et al. in northern Iran (Daryani et al. 2012). A parasite's ova from the anal region should be detected using swabs or transparent adhesive tape, so stool examination is not enough to identify the parasite. Since there is a very slight possibility of finding the parasite's ova in the stool (Abah and Arene 2015), the low prevalence of this parasite in the present study may be due to the sampling method.

G. lamblia, like other studies in Iran (Taheri et al. 2011; Tappe et al. 2011), was the most common intestinal parasite, among the studied subjects. Although Giardia, similar to many parasites, can be spread by water and possibly food, it seems that direct person-to-person transmission is the most important transmission method among school children (Daryani et al. 2012). The prevalence rate of this parasite comparing to the previous study was very low (Sharifi Sarasiabi et al. 2002) (17.23\%). Another common parasite in this study was $B$. hominis, with a prevalence similar to the study by Masoumeh et al. (2012). Since the laboratory report of this parasite is not so common, it is likely that the prevalence of this parasite may be higher than in some mentioned studies.

The present study showed significant association between parents' literacy level and jobs and the intensity of the parasitic infection, which was consistent with other studies in Iran (Nematian et al. 2004; Masoumeh et al. 2012) and other countries (Al-Mohammed et al. 2010; Ayalew et al. 2011). However, it was inconsistent with the study by Masoumeh et al. (2012). The relationship between sanitary and health conditions of children and the literacy level of parents is justifiable, but the relationship between parasitic infection and the father's job is possibly due to factors associated with economic and social conditions (Taheri et al. 2011).

Several studies have reported a higher prevalence of infection in boys than girls (Jejaw et al. 2015; Yentur Doni et al. 2015), but some have reported contrary findings (Nematian et al. 2004; Mukhiya et al. 2012; Sah et al. 2013). In the present study, the prevalence of intestinal parasites was slightly higher in boys without significant differences, which was similarly found in the study by Warunee et al. (2007). The reason is likely that the boys play outside and have contact with the soil-transmitted helminths or waterborne parasites which are transmitted easily through water (Yentur Doni et al. 2015).

There was also no significant difference between the prevalence of intestinal parasites and age, which is similar to the results reported by other studies in Iran (Taheri et al. 
2011; Daryani et al. 2012) and other countries (Korzeniewski et al. 2015).

The low prevalence of intestinal parasites in the east region of Bandar Abbas, in comparison with the west, might be due to sanitary conditions of schools and living places or possibly the overpopulation in western areas.

In this study, only one stool sample was taken from each participant for detection of intestinal parasites, while it is recommended that three samples are taken from each person. In addition, it would have been better to use a specific diagnostic method for parasites like E. vermicularis which was unfortunately not possible because of lack of cooperation of participants.

\section{Conclusion}

The prevalence of intestinal parasites, compared to the previous study in this area, was significantly lower which is mainly due to an increase in the quality of life and parents' education and also the accessibility to health services. The results of this study showed that the most common intestinal parasite was G. lamblia, and therefore it is recommended that more emphasis is applied to controlling and preventing this parasitic infection. The most important and easiest preventive method for parasitic infection of children is washing hands, particularly before each meal course.

Acknowledgements The study was carried out with the support of HUMS, Bandar Abbas, Iran. We are grateful to the officials of education and training of Bandar Abbas for the friendly cooperation and all the children and their parents for their collaboration. We are also grateful to the health practitioners and the officials of the schools.

\section{Compliance with ethical standards}

Conflict of interest The authors declare that there is no conflict of interests.

\section{References}

Abah AE, Arene FO (2015) Status of intestinal parasitic infections among primary school children in Rivers State, Nigeria. J Parasitol Res 2015:937096

Al-Mohammed HI, Amin TT, Aboulmagd E, Hablus HR, Zaza BO (2010) Prevalence of intestinal parasitic infections and its relationship with socio-demographics and hygienic habits among male primary schoolchildren in Al-Ahsa, Saudi Arabia. Asian Pac J Trop Med 3(11):906-912

Ayalew A, Debebe T, Worku A (2011) Prevalence and risk factors of intestinal parasites among Delgi school children, North Gondar, Ethiopia. J Parasitol Vector Biol 3(5):75-81

Daryani A, Sharif M, Nasrolahei M, Khalilian A, Mohammadi A, Barzegar G (2012) Epidemiological survey of the prevalence of intestinal parasites among schoolchildren in Sari, northern Iran. Trans R Soc Trop Med Hyg 106(8):455-459
Fallahi S, Rostami A, Mohammadi M, Ebrahimzadeh F, Pournia Y (2016) Practical parasitology courses and infection with intestinal parasites in students. $\mathrm{J}$ Infect Public Health. doi: 10.1016/j.jiph.2015.12.010

Gelaw A, Anagaw B, Nigussie B, Silesh B, Yirga A, Alem M, Endris M, Gelaw B (2013) Prevalence of intestinal parasitic infections and risk factors among schoolchildren at the University of Gondar Community School, Northwest Ethiopia: a crosssectional study. BMC Public Health 13:304

Gimba U, Dawam N (2015) Epidemiological status of intestinal parasitic infection rates in children attending Gwagwalada township clinic, FCT-Abuja, Nigeria. Am J Res Commun 3(2):97-110

Jejaw A, Zemene E, Alemu Y, Mengistie Z (2015) High prevalence of Schistosoma mansoni and other intestinal parasites among elementary school children in Southwest Ethiopia: a crosssectional study. BMC Public Health 15:600

Korzeniewski K, Augustynowicz A, Smolen A, Lass A (2015) Epidemiology of intestinal parasitic infections in school children in Ghazni Province, eastern Afghanistan. Pak J Med Sci 31(6):1421-1425

Mahmud MA, Spigt M, Bezabih AM, Pavon IL, Dinant GJ, Velasco GB (2015) Efficacy of handwashing with soap and nail clipping on intestinal parasitic infections in school-aged children: a factorial cluster randomized controlled trial. PLoS Med 12(6):e1001837 (Discussion e1001837)

Masoumeh R, Farideh T, Mitra S, Heshmatollah T (2012) Intestinal parasitic infection among school children in Golestan province, Iran. Pak J Biol Sci 15(23):1119

Matthys B, Bobieva M, Karimova G, Mengliboeva Z, Jean-Richard V, Hoimnazarova M, Kurbonova M, Lohourignon LK, Utzinger J, Wyss K (2011) Prevalence and risk factors of helminths and intestinal protozoa infections among children from primary schools in western Tajikistan. Parasit Vectors 4(1):195

Moore CE, Nget P, Saroeun M, Kuong S, Chanthou S, Kumar V, Bousfield R, Nader J, Bailey JW, Beeching NJ, Day NP, Parry CM (2015) Intestinal parasite infections in symptomatic children attending hospital in Siem Reap, Cambodia. PLoS ONE 10(5):e0123719

Mukhiya R, Rai S, Karki A, Prajapati A (2012) Intestinal protozoan parasitic infection among school children. J Nepal Health Res Council 10(22):204-207

Nematian J, Nematian E, Gholamrezanezhad A, Asgari AA (2004) Prevalence of intestinal parasitic infections and their relation with socio-economic factors and hygienic habits in Tehran primary school students. Acta Trop 92(3):179-186

Okyay P, Ertug S, Gultekin B, Onen O, Beser E (2004) Intestinal parasites prevalence and related factors in school children, a western city sample-Turkey. BMC Public Health 4:64

Oliveira D, Ferreira FS, Atouguia J, Fortes F, Guerra A, CentenoLima S (2015) Infection by intestinal parasites, stunting and anemia in school-aged children from Southern Angola. PLoS One 10(9):e0137327

Rokni MB (2008) The present status of human helminthic diseases in Iran. Ann Trop Med Parasitol 102(4):283-295

Sah RB, Bhattarai S, Yadav S, Baral R, Jha N, Pokharel PK (2013) A study of prevalence of intestinal parasites and associated risk factors among the school children of Itahari, Eastern Region of Nepal. Trop Parasitol 3(2):140

Sharifi Sarasiabi K, Madani A, Zare S (2002) Prevalence of intestinal parasites in primary school pupils of bandar abbas. Hormozgan Med J 4(5):25-30

Shokri A, Sarasiabi KS, Teshnizi SH, Mahmoodi H (2012) Prevalence of Strongyloides stercoralis and other intestinal parasitic infections among mentally retarded residents in central institution of southern Iran. Asian Pac J Trop Biomed 2(2):88-91 
Taheri F, Namakin K, Zarban A, Sharifzadeh G (2011) Intestinal parasitic infection among school children in South Khorasan Province, Iran. J Res Health Sci 11(1):45-50

Tappe KH, Mohammadzadeh H, Khashaveh S, Rezapour B, Barazesh A (2011) Prevalence of intestinal parasitic infections among primary school attending students in Barandooz-Chay rural region of Urmia, West Azerbaijan province, Iran in 2008. Afr J Microbiol Res 5(7):788-791
Warunee N, Choomanee L, Sataporn P, Rapeeporn Y, Nuttapong W, Sompong S, Thongdee S, Bang-On S, Rachada K (2007) Intestinal parasitic infections among school children in Thailand. Trop Biomed 24(2):83-88

Yentur Doni N, Gurses G, Simsek Z, Yildiz Zeyrek F (2015) Prevalence and associated risk factors of intestinal parasites among children of farm workers in the southeastern Anatolian region of Turkey. Ann Agric Environ Med 22(3):438-442 